\title{
Improving the description of proton-induced one-nucleon removal in intranuclear-cascade models
}

\author{
Davide Mancusi, ${ }^{1,}$ A Alain Boudard, ${ }^{1}$ Jaume Carbonell, ${ }^{2}$ Joseph Cugnon, ${ }^{3}$ Jean-Christophe David, ${ }^{1}$ and Sylvie Leray ${ }^{1}$ \\ ${ }^{1}$ CEA, Centre de Saclay, Irfu/SPhN, F-91191 Gif-sur-Yvette CEDEX, France \\ ${ }^{2}$ Institut de Physique Nucléaire, Université Paris-Sud, IN2P3-CNRS, F-91406 Orsay CEDEX, France \\ ${ }^{3}$ AGO Department, University of Liège, Allée du 6 août 17, Bât. B5, B-4000 Liège 1, Belgium
}

(Received 18 November 2014; published 3 March 2015)

\begin{abstract}
It is a well-established fact that intranuclear-cascade models generally overestimate the cross sections for one-proton removal from heavy, stable nuclei by a high-energy proton beam, but they yield reasonable predictions for one-neutron removal from the same nuclei and for one-nucleon removal from light targets. We use simple shell-model calculations to investigate the reasons for this deficiency. We find that a refined description of the neutron skin and of the energy density in the nuclear surface is crucial for the aforementioned observables, and that neither ingredient is sufficient if taken separately. As a by-product, the predictions for removal of several nucleons are also improved by the refined treatment.
\end{abstract}

DOI: 10.1103/PhysRevC.91.034602

PACS number(s): 24.10.Lx, 25.40.Sc, 21.60.Cs

\section{INTRODUCTION}

Nuclear reactions between high-energy ( $\gtrsim 150 \mathrm{MeV}$ ) nucleons or hadrons and nuclei are usually described by means of hybrid models consisting of an intranuclear-cascade (INC) stage followed by a statistical de-excitation stage [1]. In this framework, the projectile is assumed to initiate an avalanche of binary collisions with the nucleons of the target, which can lead to the emission of energetic particles. The nature of INC models is essentially classical. It is typically assumed that nucleons are perfectly localized in phase space and that they are bound by an average, constant potential; moreover, it is assumed that subsequent elementary collisions are independent.

Despite the simplicity of such reaction models, it was proved that they are able to describe a vast array of experimental observables with a very restricted number of free parameters [2]. However, it was realized some time ago that these models systematically fail to describe inclusive cross sections for the removals of few nucleons, for example, [3,4]. This is especially surprising in view of the fact that these observables are associated with peripheral reactions and mostly involve collisions between quasifree nucleons; one would therefore expect the intranuclear cascade to provide an accurate description of this particular dynamics. This puzzling result has been known for many years now, but no convincing explanation has ever been put forward.

Note that, in general, the prediction of the inclusive onenucleon-removal cross sections at high energy can reasonably be tackled only with a two-step dynamical/de-excitation model. One-nucleon removal, in fact, results from events of a specific class: (1) few nucleon-nucleon scatterings must take place, and (2) the residual excitation energies after knockout must fall within a given window. If one of these conditions is not verified, removal of several nucleons becomes the most likely outcome. The models that are usually applied to the study of knockout reactions [5,6] either do not properly account for the probability of multiple collisions (condition

*davide.mancusi@cea.fr
1) or do not account for all the relevant residual states after knockout (condition 2).

One-nucleon removal, being associated with peripheral reactions, is certainly sensitive to the details of the description of the nuclear surface, such as the density profile. Arguably, the semiclassical initial conditions of INC might be inadequate for this purpose. The aim of this paper is to investigate the possibility to accommodate some genuine quantum-mechanical features of the nuclear surface into INC, by appealing to simple shell-model calculations and by casting their results in a form adaptable to INC. We will show to what extent the predictions of a particular INC model [7] can be thus improved.

Section II gives a brief description of the INC framework, whose appropriateness for the problem at hand is specifically discussed in Sec. II B. The experimental data for one-nucleonremoval cross sections are presented and interpreted in Sec. III. Our shell-model calculations are described in Sec. IV, while the refined INC model is introduced in Sec. V. Results of the calculations with the new model are presented in Sec. VI, before the conclusions in Sec. VII.

\section{INTRANUCLEAR CASCADE}

\section{A. Model description}

Intranuclear cascade [8] is a class of models that are commonly used for the description of proton-induced reactions at high energy $(>150 \mathrm{MeV})$. In this context it is assumed that the first stage of the reaction can be described as an avalanche of independent binary collisions. The INC scheme can be derived from the usual nuclear transport equations under suitable approximations $[9,10]$ and its numerical solution can be efficiently tackled on today's computers. The INC model is essentially classical, with the addition of a few suitable ingredients that mimic genuine quantum-mechanical features of the initial conditions and of the dynamics: For instance, target nucleons are endowed with Fermi motion, realistic space densities are used, the output of binary collisions is random, and elementary nucleon-nucleon collisions are subject to Pauli blocking.

At the end of the intranuclear cascade, an excited remnant is left. This nucleus typically relaxes by emitting low-energy 
particles or, when applicable, by fissioning. The time scale for the second stage is typically much longer than that for the first one, which justifies the fact that de-excitation is not described by INC but by a different class of models which rely on statistical assumptions about the properties of the excited remnant. It is essential to couple INC to a de-excitation model if one wishes to describe the production of cold (i.e., observable) reaction residues.

INC approximates the exact dynamics of the nuclear reaction as a sequence of binary collisions. However, the initial conditions of the reaction, which typically amount to the ground state of the target nucleus and which are in principle also determined by the exact nuclear dynamics, cannot be determined within the INC approximation. It is therefore necessary to specify them as an additional model ingredient.

In what follows, we make explicit reference to the Liège Intranuclear Cascade model (INCL) [11] and the ABLA07 statistical de-excitation model [12]. The INCL/ABLA07 coupling is in general quite successful at describing a vast number of observables in nucleon-induced reactions at incident energies between $\sim 60$ and $3000 \mathrm{MeV}$ [2]. For technical reasons, the work described hereafter was performed with the latest $\mathrm{C}++$ version of the INCL code (INCL++ v5.1.14) [7]. For the matter at hand, INCL++ is essentially equivalent to the reference INCL4.6 version [11].

The INCL model is peculiar in that it explicitly tracks the motion of all the nucleons in the system, which are assumed to move freely in a square potential well. The radius of the well is not the same for all nucleons, but it is rather a function $R(T)$ of the nucleon kinetic energy (which is a conserved quantity in the absence of collisions). The initial nucleon momenta are uniformly distributed in spheres of radii,

$$
\begin{aligned}
\left.p_{F} \text { (proton }\right) & =(2 Z / A)^{1 / 3} p_{F}, \\
p_{F}(\text { neutron }) & =(2 N / A)^{1 / 3} p_{F},
\end{aligned}
$$

with $p_{F}=270 \mathrm{MeV} / c$. The relation between kinetic energy and radius of the potential well is such that the space density distribution is given by a fixed, isospin-independent, suitable Woods-Saxon parametrization [13].

As an example, we discuss the phase-space density of protons in ${ }^{208} \mathrm{~Pb}$, as defined by the INCL initial conditions. Figure 1 shows the proton kinetic-energy and space distributions as solid black lines: The kinetic-energy distribution represents a uniform sphere in momentum space, while the space distribution is the classic Woods-Saxon distribution. Figure 2(a) illustrates how the kinetic energy correlates with the radius of the potential well where the protons move. For a given kinetic energy, the density is constant up to a certain distance from the center, which is the radius of the potential well $R(T)$; beyond this radius, the density vanishes. The radius of the well increases as the kinetic energy increases and reaches the radius of the calculation sphere (inside which the simulation takes place; here about $11 \mathrm{fm}$ ) as the kinetic energy tends to the Fermi energy. The distributions shown in Fig. 1 are simply projections of Fig. 2(a) on each of the axes (up to multiplication by appropriate Jacobians).

In substance, the motion of nucleons in the INCL nucleus is such that the closer they are to the Fermi energy, the farther
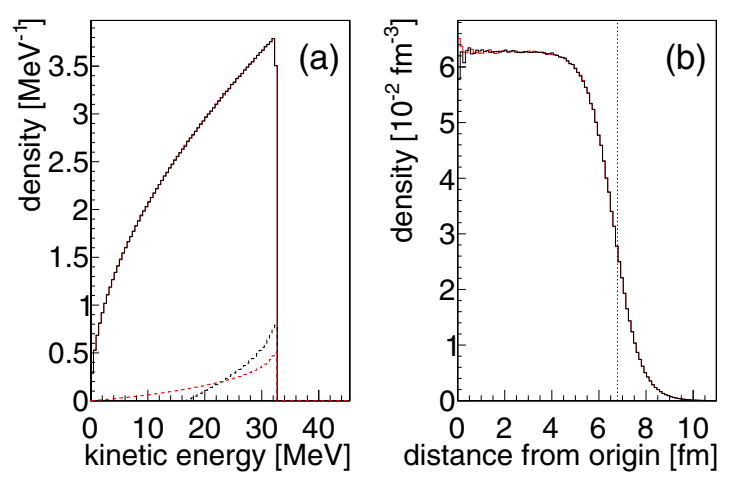

FIG. 1. (Color online) Proton kinetic-energy (a) and space (b) densities in ${ }^{208} \mathrm{~Pb}$ in the standard INCL initial conditions (solid black lines) and in the refined initial conditions with $f=0.5$ (solid red lines; see Sec. V for the definition of $f$ ). The solid black and red lines are essentially on top of each other in both panels. One-proton removal is dominated by impact parameters to the left of the vertical dotted line in (b). The dashed lines in (a) are the energy distributions of protons that are found to the right of the dotted vertical line of (b), for the standard (dashed black line) and refined (dashed red line) INCL initial conditions.

out they move in space; this trait is inspired by the properties of classical particle motion in a potential well. Therefore, the nuclear surface of the nucleus is predominantly populated by nucleons whose energy is close to the Fermi energy.

\section{B. Appropriateness of INC/de-excitation}

As we mentioned in the Introduction, dynamical/deexcitation models are the only ones that can reasonably attempt an inclusive description of one-nucleon removal at high energy. The dynamical stage may be described by
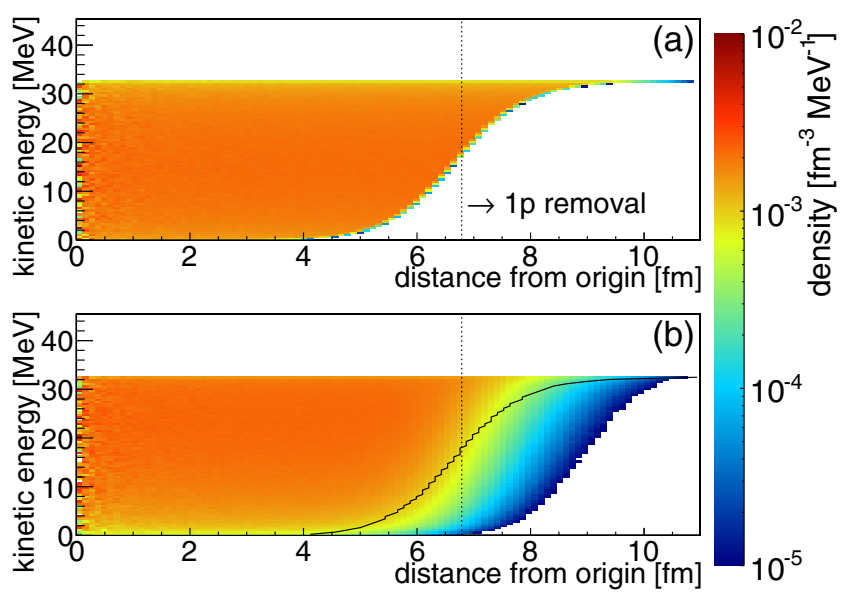

FIG. 2. (Color online) Space-kinetic-energy density of protons in ${ }^{208} \mathrm{~Pb}$ in the standard INCL initial conditions (a) and in the refined initial conditions with $f=0.5$ (b); see Sec. V for the definition of $f$. The dotted vertical lines indicate the region of impact parameters which dominates one-proton removal. The contour of the colored shape in (a) represents the inverse of the function $R(T)$ and is reported as a solid black line in (b). 

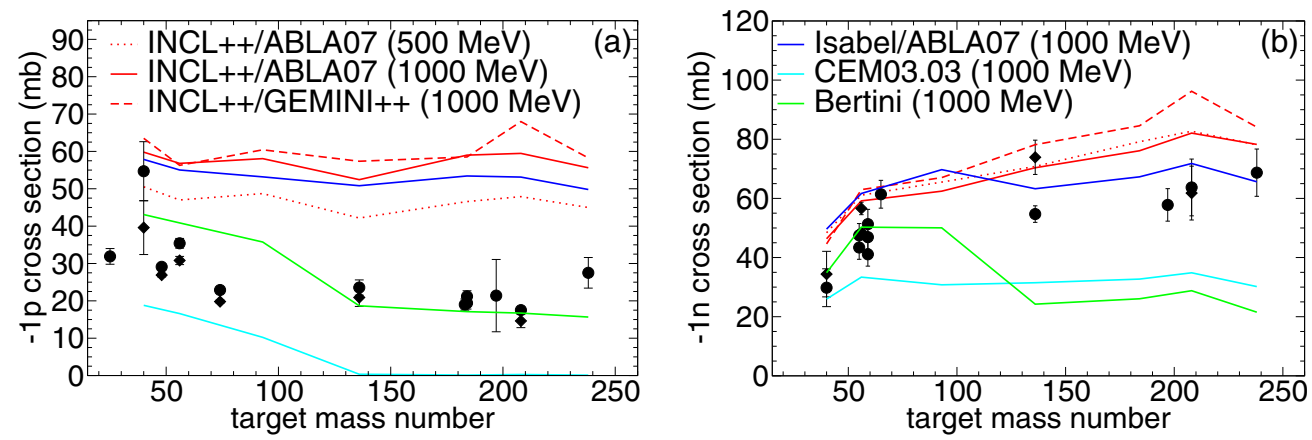

FIG. 3. (Color online) Experimental data for one-proton- (a) and one-neutron-removal cross sections (b) in proton-nucleus reactions above $500 \mathrm{MeV}$ incident energy, as a function of the target mass. Diamonds refer to experimental beam energies between 500 and $750 \mathrm{MeV}$, while circles represent energies above $750 \mathrm{MeV}$. The solid lines represent calculations with INCL/ABLA07 (red), Isabel/ABLA07 (blue), CEM03.03 (cyan), and Geant4's Bertini model (green) at $1000 \mathrm{MeV}$. The dashed red lines represent INCL/GEMINI++ calculations at $1000 \mathrm{MeV}$. The dotted red lines represent INCL/ABLA07 calculations at $500 \mathrm{MeV}$. Experimental data taken from Refs. [3,14-24].

INC (as in this paper) or by other models, such as the Boltzmann-Uehling-Uhlenbeck (BUU) or Vlasov-UehlingUhlenbeck (VUU) approaches [25], or models from the family of quantum molecular dynamics (QMD) [26-28]. These models are all "INC-like" insofar as they superimpose a cascade of binary collisions on some kind of particle dynamics. For the sake of simplicity, in what follows we will always refer to INC models, but most of our analysis can be generalized to other classes of dynamical models.

Other kinds of nuclear-reaction models, such as the distorted-wave Born approximation (DWBA) or distortedwave impulse approximation (DWIA) [5,6], are not expected to be applicable to the description of one-nucleon removal, for two main reasons.

First, one-nucleon removal must be dominated by events with few nucleon-nucleon scatterings, perhaps only one. This ensues from the fact that the average energy transfer in nucleon-nucleon scattering at high energy is large; therefore, multiple collisions are liable to lead to many-nucleon removal. The probability for multiple collisions must therefore be correctly evaluated. In DWBA and DWIA, rescattering is modeled as absorption owing to the imaginary part of an optical potential, which seems far-fetched at the energies we are concerned with. Analyses of proton spectra from the continuum have indeed shown that, even in the $200-400 \mathrm{MeV}$ incident-energy range, it is necessary to go beyond DWIA insofar as the description of rescattering is concerned [see, e.g., 29-31]. Finally, the evaluation of rescattering at higher energies is complicated by the possible production of pions in the knockout collision.

Second, the one-nucleon-removal cross sections are fed by knockout reactions leading to all residue states below the particle-separation energy. In addition, the cross section can also receive contributions from the continuum, i.e., from excitation energies above the particle-separation energy. This is especially true for neutron removal, which can proceed through the formation of an equilibrated system. The decay of the latter (and the competition between the various channels) is accounted for in INC/de-excitation, but not in DWBA/DWIA.

In any case, the INC/de-excitation (dynamical/deexcitation) approach is the only one which can tackle with the same (combined) model all residue formation channels, from one-nucleon-removal channels to deep spallation channels where a substantial fraction of the target nucleons are removed.

Admittedly, INC suffers for other limitations, the most important being the semiclassical nature of the initial conditions. This is reflected, for instance, in the lack of a discrete level structure. The INC predictions are typically smooth functions of the reaction parameters (charge, mass, energy,...). However, it is possible to allow for certain genuine quantum-mechanical aspects in an effective manner. This is what we propose to illustrate in Secs. IV and V.

\section{ONE-NUCLEON REMOVAL}

\section{A. Cross sections}

Figure 3 shows the experimental data for one-nucleon removal in proton-induced reactions at energies $\gtrsim 500 \mathrm{MeV}$, as a function of the target mass (all targets are close to the stability valley). Calculations with INCL/ABLA07 at 500 and $1000 \mathrm{MeV}$ are shown for comparison. It is clear that the model predictions are in the right ballpark for neutron removal, but they overestimate the proton-removal data by a factor that can be as large as 3-4 for heavy nuclei. Note that the experimental data are globally consistent, even though they have been collected in inverse-kinematics experiments [14-21] or by off-line gamma spectroscopy [3,22-24].

The role played by de-excitation can be clarified by comparing calculations with the same INC but different de-excitation models. Therefore, we performed calculations with INCL coupled with the GEMINI++ model [32]. The resulting cross sections (shown in Fig. 3 as dashed red lines) are within $20 \%$ of the INCL/ABLA07 values and indicate that the influence of de-excitation is somewhat mild. Therefore, it seems unlikely that de-excitation can be held responsible for the gross overestimation of proton-removal cross sections.

We also show in Fig. 3 the results of calculations that we have performed with other INC models. The results of Isabel [33], in coupling with ABLA07, are qualitatively similar to the INCL results and exhibit the same defect for proton removal. Geant4's Bertini-like cascade [34] is quite different inasmuch as it yields somewhat correct predictions for proton 
removal, but it badly underestimates neutron removal. Finally, CEM03.03 [35] yields very low proton-removal cross sections (of the order of $0.1 \mathrm{mb}$ for $A>130$ ) and underestimates neutron removal by roughly a factor of two.

The calculations presented in Fig. 3 globally demonstrate that INC models have difficulty in correctly predicting the inclusive one-nucleon-removal cross sections. No model is able to describe proton- and neutron-removal cross sections on all targets. This is somewhat surprising on two counts. First, one-nucleon-removal cross sections are among the largest isotopic cross sections, they are only modestly influenced by de-excitation, and they vary slowly with the target mass and the projectile energy; thus, they represent an excellent test bench for INC models, but they seem to have attracted little attention so far. Second, one-nucleon-removal reactions are typically dominated by very peripheral impact parameters, which probe regions of the nucleus with large mean free path (low density); in addition, the collision partners are somewhat localized in the nuclear surface, i.e., they are loosely bound. One would expect the INC approximation to be fully justified under these conditions. The failure illustrated by Fig. 3 suggests that INC models might be affected by a fundamental defect.

There are other remarkable features of the INC failure. One might expect even more conspicuous mispredictions for the removal of a larger number of nucleons, but one instead finds that the models can generally reproduce most of the isotopic distributions somewhat well (see the isotopic distributions in Sec. VI and Ref. 2). This should be understood as a consequence of the larger excitation energies associated with the emission of several nucleons. Because large excitation energies can be realized in numerous ways, some averaging takes place and the predictions become less sensitive to the details of the initial conditions. At the same time, it should be stressed that discrepancies do seem to increase for the removal of, e.g., several protons from stable nuclei [4]. This is consistent, inasmuch as the constraints on the excitation energy in that case are even stricter than for one-proton removal, as evidenced by the smallness of the associated cross sections.

Note that the experimental cross sections do not seem very sensitive to the reaction parameters, such as the beam energy and the target species. This suggests that the details of the level structure of the individual nuclides involved do not play an important role. Therefore, it might be possible to amend INC and describe these observables, but it is probably necessary to go beyond the naive semiclassical model of the nuclear surface. We will do so in Secs. IV and V, but we first need to clarify the mechanism that leads to proton and neutron removal within the INC framework.

\section{B. Removal mechanism}

Let us first concentrate our attention on proton removal. The analysis of the INC/de-excitation calculations indicates that proton removal is dominated (about $90 \%$ of the cross section) by events with only one proton-proton collision. The two protons leave the nucleus, which, however, retains some excitation energy. If only one collision takes place, the excitation energy is simply given by the depth of the proton hole, i.e., the difference between the Fermi energy and the initial energy of the ejected proton. In any case, the excitation energy remaining at the end of the cascade is evacuated during the de-excitation phase.

Note that, for most $\beta$-stable, nonfissile nuclei, particle emission at low excitation energy is largely dominated by neutron evaporation (for the sake of illustration we neglect light nuclei, for which proton and $\alpha$ evaporation can become competitive against neutron evaporation). If the excitation energy is lower than the neutron separation energy $S_{n}$, no particle can be evaporated and the energy will be evacuated as gamma rays. This is also true at energies slightly larger than $S_{n}$, as long as gamma-ray emission outcompetes neutron evaporation; thus, the effective neutron-evaporation threshold $S_{n}^{*}$ is slightly larger than $S_{n}$. Therefore, the proton-removal channel is populated if and only if exactly one proton was ejected during INC and the excitation energies at the end of the cascade lie below $S_{n}^{*}$. If the excitation energy allows for neutron evaporation, the final residue will be lighter (target minus one proton minus $x$ neutrons).

The observations above highlight two important aspects. First, the proton-removal cross section is extremely sensitive to the excitation energy left in the nucleus after the ejection of a proton during INC. More precisely, the cross section is determined by the probability that the ejection of a proton during INC deposits an excitation energy smaller than $S_{n}^{*}$. Second, there is a subtle difference between proton and neutron removal. Neutron removal can be realized in two ways: either as a neutron ejection during INC followed by no evaporation (this is analogous to the proton-removal mechanism), or as no neutron ejection during INC followed by evaporation of one neutron. In the latter scenario it is of course required that the incoming proton undergoes at least one binary collision and that it succeeds in escaping from the target; some conditions on the excitation energy also apply.

In either case, the fate of the de-excitation stage is essentially determined by the excitation energy at the end of INC and by the neutron separation energies in the region of the nuclide chart around the target. In this sense, our results are essentially independent of the choice of the de-excitation model, as far as all of them employ very similar separation energies for stable nuclei. The second-order dependence on the de-excitation model (see the ABLA07/GEMINI++ difference) can be ascribed to differences in the neutron-gamma competition, i.e., by slightly different values of the effective neutron-evaporation thresholds $S_{n}^{*}$.

It is clear then that the smallness of the excitation energy at the end of INC, especially in the proton-removal case, is the crucial element that determines one-nucleon-removal cross sections. Comparison with the experimental data (Fig. 3) seems to suggest that INCL largely underestimates the excitation energy associated with the ejection of a proton. Similar remarks have been made about "cold fragmentation" in peripheral nucleus-nucleus reactions [36]. It was found that the excitation energy predicted by the abrasion model needs to be multiplied by roughly a factor of two to explain the cross sections for the removal of one or more protons. Given the simple nature of the abrasion model, however, the generality of this conclusion is unclear. 
Some important remarks are due at this point. Although INCL and Isabel are in quantitative disagreement with the experimental data (Fig. 3), they correctly capture the overall dependence on the target mass. Bertini and CEM03.03, on the other hand, yield trends that are sensibly different from the experimental ones. This seems to point to the existence of two classes of models and might be correlated with the presence of an intermediate pre-equilibrium stage in Bertini and CEM03.03. Because one-nucleon removal is essentially a surface process, it is somewhat sensitive to the geometrical arrangement of the first nucleon-nucleon collision. Treating one of the collision partners as a pre-equilibrium exciton amounts to discarding all information about its localization in configuration space. This probably entails lower emission probabilities and higher excitation energies compared to a full INC treatment, and might explain CEM03.03's low proton-removal cross sections.

For completeness sake, one should also remark that the two classes of models may also be characterized by the nature of the INC stage. On the one hand, Bertini and CEM03.03 are "spacelike" INC models, i.e., they sequentially track cascading nucleons until either they escape or their energy falls below a given level. On the other hand, INCL and Isabel are "timelike" INC models, i.e., they simultaneously track all cascading nucleons according to a global clock. Nevertheless, one would not expect important differences between these two approaches for reactions involving such a small number of cascading nucleons. It is doubtful to us that this element can explain the contrasting cross-section trends.

Finally, note that there are other ingredients which could in principle affect the one-nucleon-removal cross sections, such as the cross section of the first nucleon-nucleon collision, its kinematics, the parameters of the nuclear-density function (radius and diffuseness), the value of the Fermi momentum, the depth of the nucleon potential well, the height of the Coulomb barrier assumed in INC and in de-excitation, and the separation energies during the INC stage. We have verified that reasonable changes in these ingredients either have a negligible effect on the calculated one-nucleon-removal cross sections or degrade the agreement for neutron removal.

It is worthwhile at this point to summarize the statement of the problem and our motivation. One has a somewhat successful semiclassical model for spallation reactions, which can describe with the same ingredients channels resulting from the ejection of few particles, as well as those corresponding to the emission of a substantial part of target nucleons. This model seems to clearly fail on a few channels, basically the one-nucleon-removal channels. We gave arguments indicating that this is from the fact that these channels correspond to a single-scattering mechanism leaving the target with a small excitation. We pointed out that the semiclassical nature of the model is too crude to give proper control of this excitation energy. In the following, we will illustrate a method to cure the deficiencies of INC on this point.

\section{SHELL-MODEL STUDY OF THE NUCLEAR SURFACE}

We mentioned at the end of Sec. II A that the nuclear surface in the INCL initial conditions is predominantly populated by nucleons whose energy is close to the Fermi energy. The ejection of one such nucleon during INC results in little excitation energy for the cascade remnant. The considerations in the previous section cast some suspicion upon this aspect.

In the quantum-mechanical square-well problem, the density outside the well does not vanish, even for states close to the bottom of the well. This means that there is a nonzero probability to find deeply bound particles outside the well, and eject them. This genuine quantum phenomenon is missing in the naive INC nuclear picture, as illustrated by Fig. 2(a). However, a word of caution is due. In a purely quantummechanical treatment, the surface diffuseness is at least partly from the penetration of the nucleon wave functions into the classically forbidden region; in spite of this, the INC initial conditions typically do account for a realistic diffuseness of the nuclear surface (e.g., the space density of the INCL model is a realistic Woods-Saxon distribution), although this is entirely enforced by a classical correlation between the particle position and energy. The failure of the INC initial conditions is therefore more subtle. It does not concern the presence of the tail of the spatial density but rather its energy density.

Another detail that is usually neglected in the INC picture is the presence of neutron (or proton) skins in certain nuclei. It is, for instance, somewhat well ascertained that ${ }^{208} \mathrm{~Pb}$ exhibits a neutron skin thickness (defined as the difference of the neutron and proton root-mean-square radii) of about $0.2 \mathrm{fm}$ [37]. For grazing collisions, this means that the local neutron density is several times larger than the proton density, leading to an enhanced probability for collisions on neutrons.

We have estimated the magnitude of both the effects above with a simple shell-model calculation. We assumed a central Woods-Saxon nuclear potential with a spin-orbit term and a Coulomb term for the protons [38]. We numerically solved the radial part of the Schrödinger equation and determined the radial eigenfunctions $R_{n j}^{i}(r)$ and the eigenvalues $E_{n j}^{i}$ of the bound states (here $i=p, n$ ). The single-particle energies $E_{n j}^{i}$ in ${ }^{208} \mathrm{~Pb}$ correctly reproduce the energies of the lowest-lying particle-hole states in ${ }^{207,209} \mathrm{~Pb}$ and ${ }^{207} \mathrm{Tl},{ }^{209} \mathrm{Bi}$.

\section{A. Space densities}

To keep the notation simple, we drop the $i$ superscript from our formulas and we consistently refer to protons (the formulas for neutrons can be straightforwardly recovered). We assume that the shells are filled from the bottom of the well up to the Fermi level. The latter may be partly empty if the nucleus is not proton-magic. The occupation numbers $g_{n j}$ are given by

$$
g_{n j}=\left\{\begin{array}{lll}
2 j+1 & \text { if } & E_{n j}<E_{\mathrm{F}} \\
Z-\sum_{E_{n j}<E_{\mathrm{F}}} g_{n j} & \text { if } & E_{n j}=E_{F} . \\
0 & \text { if } & E_{n j}>E_{F}
\end{array}\right.
$$

Here $E_{\mathrm{F}}$ denotes the Fermi energy. We construct the radial density profiles,

$$
\rho_{n j}(r)=4 \pi r^{2}\left|R_{n j}(r)\right|^{2},
$$

and the densities,

$$
\rho(r)=\sum_{n j} g_{n j} \rho_{n j}(r)
$$




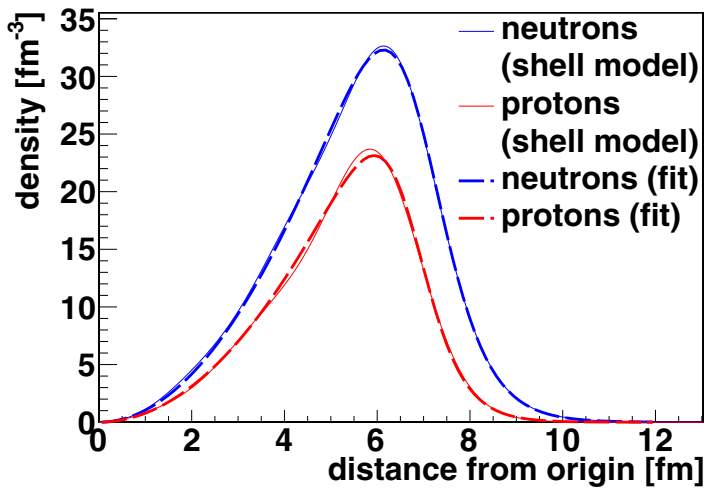

FIG. 4. (Color online) Proton (red) and neutron (blue) densities for ${ }^{208} \mathrm{~Pb}$. The thin solid lines represent the result of the shell-model calculation, while the thick dashed lines are Woods-Saxon fits. The fit parameters are given in Table I. All curves include the Jacobian factor $4 \pi r^{2}$.

The resulting proton and neutron densities are shown in Fig. 4. At each position we can also construct the presence probabilities,

$$
p_{n j}(r)=g_{n j} \rho_{n j}(r) / \rho(r) .
$$

We would like to use the shell-model proton and neutron densities as inputs for our INC calculation; however, the particle densities in INCL cannot be given by an arbitrary function, so we must somehow adapt the shell-model densities. We choose to fit them with Woods-Saxon distributions (shown in Fig. 4 as dashed lines). The best-fit parameters are indicated in Table I and show that the shell-model densities for ${ }^{208} \mathrm{~Pb}$ exhibit a neutron skin, although its thickness is slightly larger than the experimentally accepted value; this is a well-known defect of mean-field calculations [39]. The calculations for ${ }^{40} \mathrm{Ca}$ instead yield a thin proton skin.

We thus decouple the INCL parameters describing the neutron space density from those describing the proton space density. We choose not to modify the proton densities (because they are already given by fits to the experimental charge radii), but we adjust the neutron parameters by the amounts indicated in the last column of Table I.

\section{B. Energy density of the nuclear surface}

We have explained in the previous section that the outcome of single-collision cascades is sensitive to the energy of the ejected nucleon. For a given position, the shell model provides

TABLE I. Optimal parameters for Woods-Saxon densities fitting the results of shell-model calculations. The "skin/halo" values are differences of the neutron and proton parameters. All values are in fm.

\begin{tabular}{ccccc}
\hline \hline & & Neutrons & Protons & Skin/halo \\
\hline${ }^{40} \mathrm{Ca}$ & $R_{0}$ & 3.57 & 3.64 & -0.08 \\
& $a$ & 0.49 & 0.51 & -0.02 \\
${ }^{208} \mathrm{~Pb}$ & $R_{0}$ & 6.98 & 6.71 & 0.26 \\
& $a$ & 0.55 & 0.46 & 0.09 \\
\hline \hline
\end{tabular}

a decomposition of the local density in terms of the various shells [Eq. (1)].

To estimate the energy density of the surface, we assume that the probability that a collision ejects a nucleon from a given shell is proportional to the local density of the shell orbital. Furthermore, we neglect rearrangement of the other nucleons in the Fermi sea after the collision; this amounts to assuming that the excitation energy of the hole is simply given by the depth of the hole:

$$
E_{n j}^{*}=E_{\mathrm{F}}-E_{n j}
$$

where $n j$ are the quantum numbers of the hole. Putting all the pieces together, we assume that a collision at position $r$ creates a hole of excitation energy $E_{n j}^{*}\left[\right.$ Eq. (2)] with probability $p_{n j}(r)$ [Eq. (1)].

We can characterize the properties of the nuclear surface by studying the probability that the excitation energy associated with the hole does not exceed the neutron separation energy, which reads

$$
P_{E^{*}<S_{n}}(r)=\sum_{n j} p_{n j}(r) \Theta\left(S_{n}-E_{n j}^{*}\right),
$$
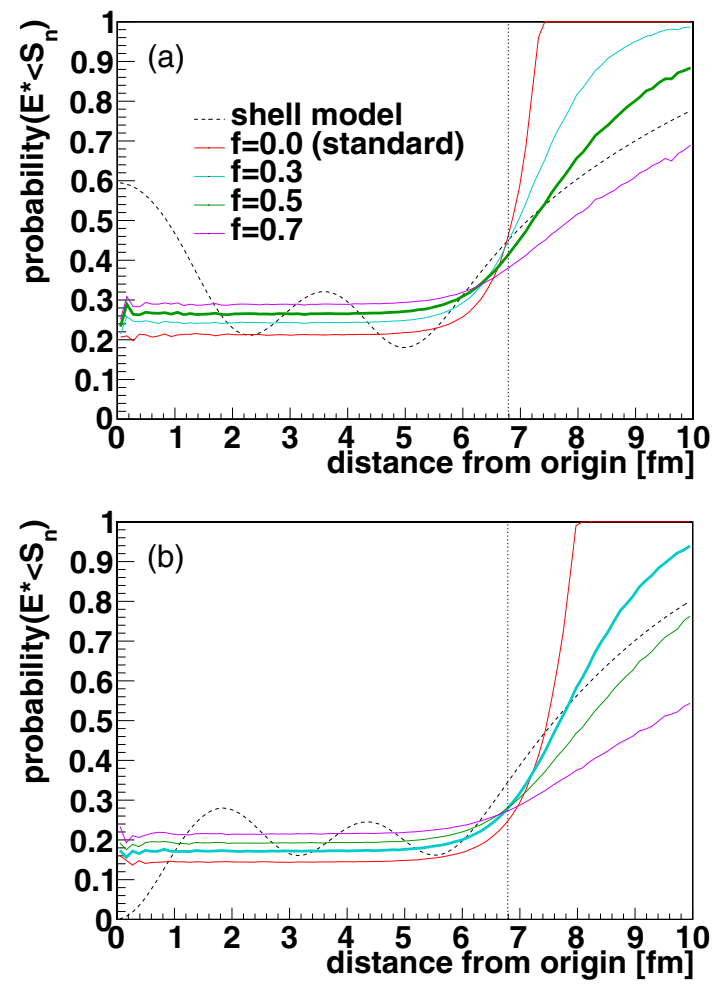

FIG. 5. (Color online) Probability that a proton (a) or neutron (b) hole in ${ }^{208} \mathrm{~Pb}$ results in an excitation energy smaller than the shell-model neutron separation energy, as a function of the distance of the hole from the center of the nucleus. The dashed line denotes the result of the shell-model calculation. Solid lines represent the INCL initial conditions for different values of the fuzziness parameter $f$ (defined in Sec. V). The standard condition corresponds to $f=0$. One-proton removal is dominated by impact parameters to the left of the vertical dotted lines (see text). 
where $\Theta$ is the Heaviside function. From our discussion in Sec. III B it should be clear that this quantity has a very important bearing on one-nucleon-removal cross sections.

The probabilities for the standard INCL initial conditions are plotted in Fig. 5 as red solid lines, as functions of the distance of the hole from the center of the nucleus. Note that the probability for shallow $\left(E^{*}<S_{n}\right.$ ) holes becomes equal to 1 beyond a certain radius. As illustrated by Fig. 2(a), this is from the fact that there is a strict minimum kinetic energy for nucleons that are found beyond a given radius.

Analysis of the INC shows that the impact-parameter distribution of events with only one INC collision peaks around $7.78 \mathrm{fm}$ and has a root-mean-square (rms) deviation of $0.99 \mathrm{fm}$. The dotted lines in Fig. 5 are set at $r=(7.78-$ $0.99) \mathrm{fm}=6.79 \mathrm{fm}$ and are meant as a guide to the eye. Roughly speaking, most single-collision reactions take place to the right of the dotted lines. The same lines are also drawn on Figs. 1(b) and 2.

It is clear from the results displayed in Fig. 5 that the standard INCL initial conditions are quite different from the results of the shell-model calculation: In the surface region, the standard INCL probability to punch a shallow hole in the Fermi sea is sensibly larger than its shell-model counterparts, which seems to confirm that the excitation energy associated with the ejection of a proton is underestimated by INCL.

\section{REFINEMENT OF THE INITIAL CONDITIONS}

We mentioned in Sec. II A that an INCL nucleon moves in a square-well potential whose radius $R(T)$ depends on the nucleon kinetic energy. The function $R(T)$ is uniquely determined by the choice of the space density $\rho(r)$ and by the assumption that nucleon momenta are uniformly distributed in the Fermi sphere. We have shown above that this construction results in excitation energies for one-collision reactions that are much smaller than those resulting from the shell model and, arguably, than those suggested by the available experimental data.

We refine the INCL initial conditions by allowing fluctuations in $R(T)$. We introduce a fuzziness parameter $f$ $(0 \leqslant f \leqslant 1)$ and a fuzzy square-well radius $R(T ; f)$. The precise definition of $R(T ; f)$ is reported in the appendix, so we limit our exposition to its most important properties: First, $R(T ; f)$ is a random variable. Second, for $f=0$ fluctuations are suppressed and we recover the standard sharp correlation:

$$
R(T ; 0)=R(T) .
$$

Third, for a given value of $T$, fluctuations in $R(T ; f)$ are small if $f$ is close to zero and they are large if $f$ is close to one. Fourth, the fluctuations are constructed in such a way that the space density is still given by $\rho(r)$ and the momentum density is still given by a uniform Fermi sphere. The construction of the fuzzy INCL nucleus is analogous to the standard preparation algorithm [13]. The only difference is that the radius of the square-well potential is no longer in one-to-one correspondence with the nucleon energy.

The phase-space structure of the fuzzy initial conditions is illustrated by Fig. 2(b), which refers to protons in ${ }^{208} \mathrm{~Pb}$ with fuzziness parameter $f=0.5$. Contrary to Fig. 2(a), we see that the density does not drop sharply to zero. Instead, protons of a given kinetic energy can sometimes be found at much larger distances than in the standard initial conditions [Fig. 2(a)]. The kinetic-energy and space distributions, i.e., the projections of Fig. 2(b), are shown in Fig. 1 as red lines. By construction, the kinetic-energy and space distributions are almost indistinguishable from those of the standard initial conditions. The fluctuations in one of the variables disappear when integrating over the full domain of the other one. Note, however, that different results are obtained if one limits the integration domain to some subrange. This is illustrated by the dashed lines in Fig. 1(a), which represent the kinetic-energy distributions of protons found "in the surface," i.e., to the right of the vertical dotted line in Fig. 1(b). The distribution of the standard initial conditions vanishes below a certain energy, while the fuzzy initial conditions extend much deeper in the Fermi sea.

The fuzzy initial conditions introduce additional energy fluctuations for the nucleons found at a given position. Figure 5 indeed demonstrates that the probability of punching a shallow surface hole decreases for increasing fuzziness, i.e., for increasing fluctuations. No value of the fuzziness parameter yields a good fit to the shell-model result, even if one limits oneself to the surface region. There is some degree of subjectivity in the choice of the best-fit values, which are taken to be $f=0.5$ for protons and $f=0.3$ for neutrons. For ${ }^{40} \mathrm{Ca}$ (not shown), the best-fit value was taken to be $f=0.3$ for both protons and neutrons.

Summarizing, we have refined the INCL initial conditions in two respects. First, we have included the possibility of introducing a neutron skin, as described in Sec. IV A. Second, we have introduced fuzzy initial conditions, which increases energy fluctuations in the nuclear surface and boosts the probability for deep-nucleon removal in surface collisions. In the framework of the shell model, this effect is genuinely quantum mechanical and it is from the penetration of the wave function in the classically forbidden region.

\section{RESULTS}

We turn now to the analysis of the results of the refined INC model. Table II shows how the neutron skin and the surface fuzziness affect the one-nucleon-removal cross sections in $p+{ }^{40} \mathrm{Ca}$ and $p+{ }^{208} \mathrm{~Pb}$. Unfortunately, no experimental data are available for $p+{ }^{40} \mathrm{Ca}$ at $1 \mathrm{GeV}$. There is one experiment at $763 \mathrm{MeV}$ by Chen et al. [14], but the resulting cross sections $\left[\sigma_{-1 p}=(54.7 \pm 7.9) \mathrm{mb} ; \sigma_{-1 n}=(29.8 \pm 6.4) \mathrm{mb}\right]$ are in sensible disagreement with the cross sections measured by the same group at lower energies and with the values suggested by the systematics of Fig. 3. Therefore, we compare our calculations with the values measured by the same group at the next lowest energy, $565 \mathrm{MeV}$.

Several observations are due. First, the introduction of the neutron skin in ${ }^{208} \mathrm{~Pb}$ boosts the neutron-removal cross section, as expected. This is, however, undesired because the cross section calculated by standard INCL is already in moderate excess of the experimental value. Second, surface fuzziness suppresses the cross sections for both channels. This is true both for ${ }^{40} \mathrm{Ca}$ and ${ }^{208} \mathrm{~Pb}$. Third, neither effect is sufficient to 
TABLE II. Cross sections for one-nucleon removal in $p$-nucleus reactions, with the following model variants: (a) standard, (b) standard plus skin, (c) standard plus surface fuzziness, (d) standard plus skin and surface fuzziness. All values are in mb. Experimental data are taken from Refs. [14,20]. Note that the small proton skin in ${ }^{40} \mathrm{Ca}$ has very little impact on the results $[(a) \simeq(b)$ and $(c) \simeq(d)]$.

\begin{tabular}{|c|c|c|c|c|}
\hline & \multicolumn{2}{|c|}{$565-\mathrm{MeV} p+{ }^{40} \mathrm{Ca}$} & \multicolumn{2}{|c|}{$1-\mathrm{GeV} p+{ }^{208} \mathrm{~Pb}$} \\
\hline & $-1 p$ & $-1 n$ & $-1 p$ & $-1 n$ \\
\hline (a) & 54.6 & 46.4 & 59.5 & 82.1 \\
\hline (b) & 54.6 & 44.6 & 50.9 & 112.0 \\
\hline (c) & 47.6 & 40.5 & 42.1 & 63.4 \\
\hline (d) & 47.3 & 38.2 & 33.6 & 83.8 \\
\hline \multirow[t]{2}{*}{ Expt. } & 39.6 & 34.4 & 17.6 & 63.7 \\
\hline & \pm 7.2 & \pm 7.7 & \pm 0.5 & \pm 9.6 \\
\hline
\end{tabular}

compensate for the overestimation of the proton-removal cross section in ${ }^{208} \mathrm{~Pb}$ if considered alone.

When the two refinements are simultaneously applied to ${ }^{208} \mathrm{~Pb}$, the effect of surface fuzziness for neutron removal almost exactly compensates the effect of the neutron skin, and the final result $(83.8 \mathrm{mb})$ is very close to the value calculated with standard INCL $(82.1 \mathrm{mb})$, which is within two standard deviations (about 30\%) of the experimental value. The proton-removal cross section, on the other hand, is reduced by almost a factor of two, which brings it much closer to the experimental datum, but not quite in agreement with it.

The results for ${ }^{40} \mathrm{Ca}$ are qualitatively similar. We observe that the cross sections are essentially insensitive to the addition of the very thin proton skin; surface fuzziness, on the other hand, reduces both cross sections by roughly the same amount (about 15\%-20\%) and brings them in better agreement with the experimental data.

The excitation curves for one-nucleon removal are shown in Fig. 6. The refined predictions are globally similar to the standard ones for neutron removal; for proton removal, the

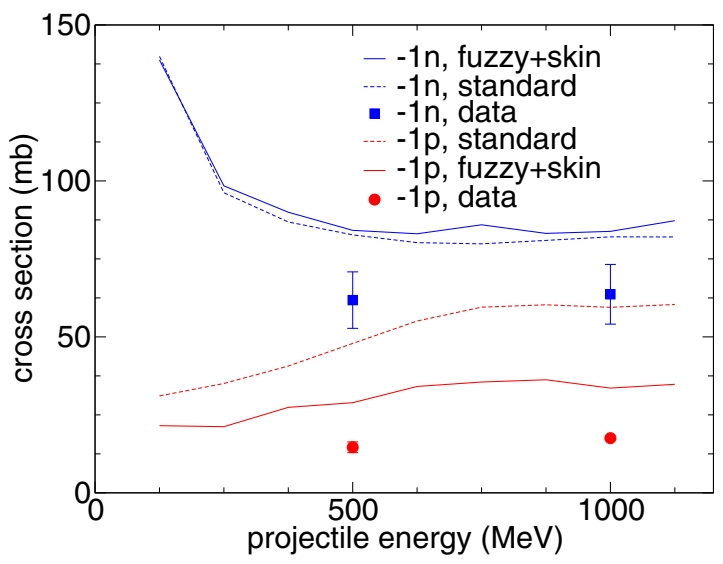

FIG. 6. (Color online) Excitation function for neutron- (blue) and proton-removal (red lines) cross sections in $p+{ }^{208} \mathrm{~Pb}$, as calculated with the standard INCL version (dashed lines) and with the refined treatment of the nuclear surface (solid lines). Experimental data are taken from Refs. [19,20].

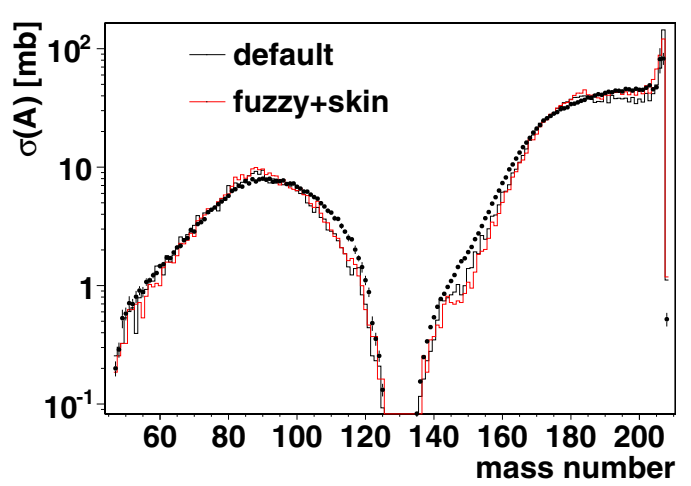

FIG. 7. (Color online) Mass distribution of the residues produced in $1-\mathrm{GeV} p+{ }^{208} \mathrm{~Pb}$. The standard INCL calculation (black) is compared to the refined calculation (red) and to the experimental data $[20,40]$.

excitation function is roughly rescaled as a whole by a factor of $\sim 0.6$. This brings the prediction in better agreement with the trend shown by the experimental data.

The global effect of neutron skin and surface fuzziness is partially illustrated by Fig. 7, which shows the mass distribution of the residues produced in $1-\mathrm{GeV} p+{ }^{208} \mathrm{~Pb}$. It is clear that, except for the $A>170$ region, the refined INCL calculation is globally very similar to the standard result. The fact that the fission peak is essentially unmodified suggests that neutron skin and surface fuzziness globally do not influence much the nature of the cascade remnants. Nevertheless, the refined treatment sensibly ameliorates the cross sections for the heaviest residues $(A>170)$, which were slightly too low in standard INCL.

Insight can be gained by examining the isotopic distributions for the heaviest residues, which are depicted in Fig. 8. Apart from the cross sections for one-proton $\left({ }^{207} \mathrm{Tl}\right)$ and one-neutron removal $\left({ }^{207} \mathrm{~Pb}\right)$, the largest differences between the standard and the refined calculations concern the isotopes of $\mathrm{Pb}$ and $\mathrm{Bi}$, which are highlighted (in linear

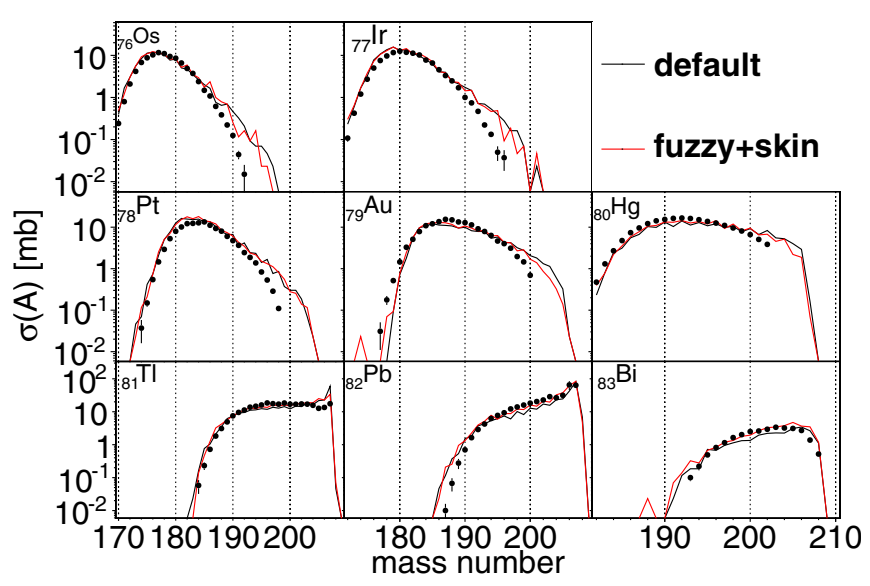

FIG. 8. (Color online) Isotopic distributions of the $Z=76-83$ residues produced in $1-\mathrm{GeV} p+{ }^{208} \mathrm{~Pb}$. The standard INCL calculation (black) is compared to the refined calculation (red) and to the experimental data [20,40]. 

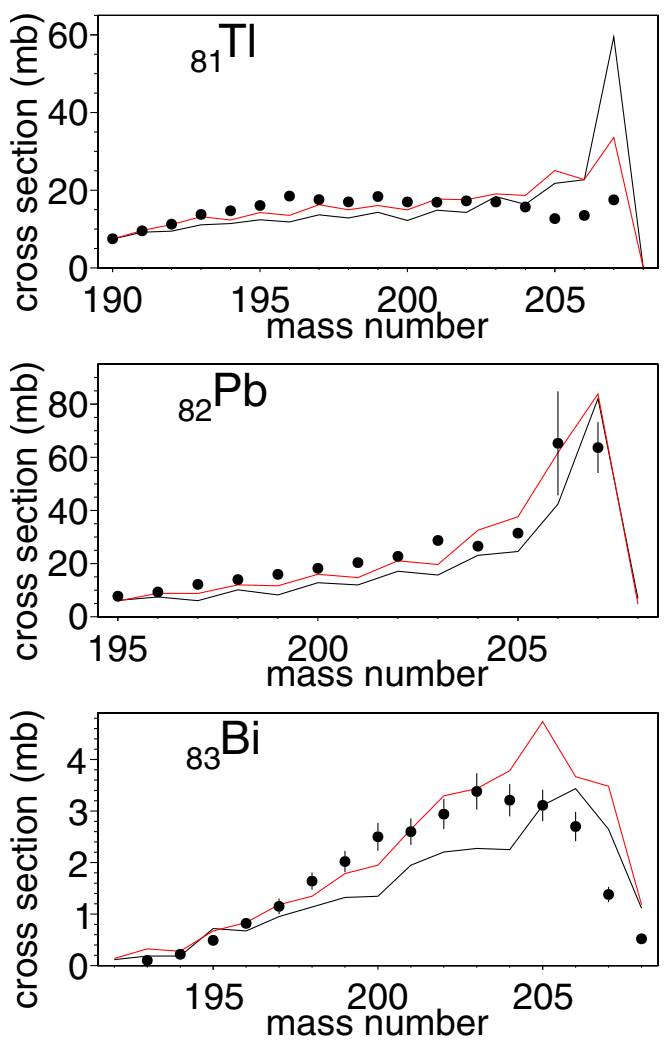

FIG. 9. (Color online) Same as Fig. 8 , for $Z=81-83$, in the linear scale.

scale) in Fig. 9. Lead and bismuth isotopes are, respectively, fed by reactions such as $(p, p x n)$ and $(p, x n)$, although the contribution from pionic channels [such as $\left(p, \pi^{+} x n\right)^{209-x} \mathrm{~Pb}$ ] is in general not negligible at all. The refined treatment of the surface considerably improves the predictions for these cross sections. Somewhat surprisingly, the cross sections for the heaviest measured Bi residues $(A=205-208)$ are degraded. Note, however, that the production of these residues imposes constraints on the cascade outcome that are even stricter than for one-nucleon removal: The excitation energy deposited in the cascade remnant must be very small, but in addition the incoming proton must be absorbed. This results in cross sections $(\sim \mathrm{mb})$ which are much smaller than those for onenucleon removal $(\sim 50 \mathrm{mb})$ and which are even more sensitive to the details of the initial conditions and of the dynamics. Generally, however, surface fuzziness and neutron skin considerably improve the cross sections for $\mathrm{Pb}$ and $\mathrm{Bi}$ isotopes. This should be seen as a by-product of the model refinement which strengthens our confidence in the new treatment.

The new description of the surface might also influence other observables; for instance, one might expect an effect on the emission patterns of particles from peripheral collisions. This is illustrated by the double-differential cross sections for neutron production from 1.2-GeV $p+\mathrm{Pb}$ shown in Fig. 10. To highlight the effect of the new surface description, we only show angles below $30^{\circ}$ and energies above $800 \mathrm{MeV}$ [the effect is much smaller in the rest of the neutron momentum space; in general, it is also smaller for outgoing protons than for outgoing neutrons (not shown)]. The peak at the

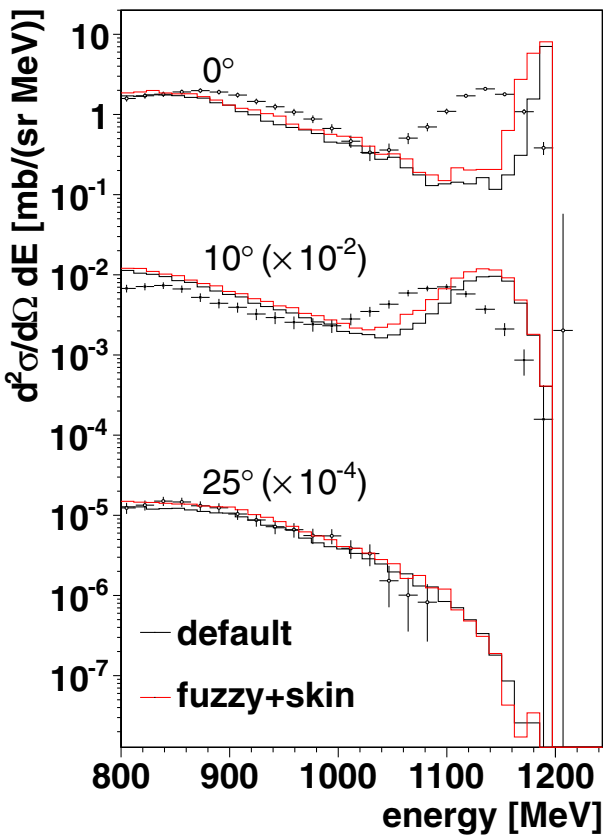

FIG. 10. (Color online) Double-differential cross sections for the production of neutrons in $1.2-\mathrm{GeV} p+\mathrm{Pb}$, as calculated with the standard INCL version (black lines) and with the refined treatment of the nuclear surface (red lines). Experimental data are taken from Ref. [41].

high-energy end of the $0^{\circ}$ and $10^{\circ}$ spectra is from quasielastic charge-exchange scattering of the incoming proton off a neutron in the target. The refined treatment of the surface leads to a broadening of the quasielastic peak, which can easily be understood as a consequence of the increased energy fluctuations of the target surface nucleons. However, the effect is minor, and it is surely insufficient to reconcile the calculation with the experimental data. This well-known disagreement has been known for quite some time [13] and is probably not specific to the INCL model. Note, however, that the shape and position of the quasielastic peak are sensitive at least to the beam profile, the beam energy distribution, the target thickness, and the detector angular acceptance. None of these aspects is realistically modeled in our calculations.

In summary, we have seen that the proton-removal cross section in $1-\mathrm{GeV} p+{ }^{208} \mathrm{~Pb}$ can be reduced by about a factor of two by taking into account the presence of the neutron skin and the surface fuzziness. However, the refined value is still in excess of the experimental one by another factor of two. One might wonder if the results we have obtained can be significantly improved by refining the calculation of the wave functions and of the energy levels (by using, e.g., the HartreeFock or Hartree-Fock-Bogoliubov methods) [42]. In keeping with the approach described above, we would then need to fit the refined probability curves with our fuzziness parameter. However, we have performed a phenomenological exploration of the parameter space and we have verified that our choice is close to optimal. It is discouraging to learn that little can be gained by refining the wave functions. Remember, however, that even the optimal fuzziness values do not reproduce the 
shell-model calculations very well (Fig. 5). In this sense there is probably margin for improvement in further refinement of the INCL initial conditions, which are manifestly not flexible enough to fit the shell-model calculations.

We wish to add a short comment about the universality of the failure of INC to appropriately describe one-nucleonremoval cross sections. The introduction of a neutron skin can be seen as a straightforward extension of the standard INC initial conditions; however, we have shown that it is not sufficient to improve the one-nucleon-removal cross sections. Surface fuzziness, on the other hand, goes definitely beyond the standard INC initial conditions. The reduction of the cross sections is ultimately from the increase of the excitation energy associated with the knock-out of surface nucleons during the INC phase. Equivalently, surface nucleons in standard INCL are too close to the Fermi energy to result in deep holes. The larger is the nucleon energy; the largest is the volume spanned by the trajectory: This assumption is absolutely natural because it draws from the behavior of classical particles. It is therefore likely to figure in all INC models where the motion of the individual target nucleons is explicitly followed.

The discussion above does not apply to intranuclear cascades that model the Fermi sea as a continuous medium (e.g., Isabel). In this case, however, collisions in low-density regions are dealt with by assuming a reduced value of the Fermi momentum (so-called local Fermi momentum) on top of a reduced depth of the potential well. Insofar as the depth of the holes that can be punched in the surface is concerned, the net result is the same: The holes lie quite close to the Fermi surface and yield somewhat small excitation energies. In this sense, the failure of INC can be described as universal, i.e., independent of the specific model incarnation.

Reaction models such as Bertini and CEM03.03 require further discussion. We have seen that they are also unable to consistently reproduce the experimental data (Fig. 3), but the disagreement is qualitatively different from models such as INCL and Isabel. There are prominent differences between these classes of models: most notably, (i) Bertini and CEM03.03 are "spacelike" INCs, while INCL and Isabel are "timelike" INCs; and (ii) Bertini and CEM03.03 include an intermediate pre-equilibrium stage, whereas INCL and Isabel are directly coupled to statistical de-excitation. It is tempting to ascribe the different behavior of the two model classes to one or both of these elements. We suggest that the use of preequilibrium might be responsible for the different behavior. As we discussed above, pre-equilibrium models carry no information about the localization of excitons in configuration space. Of course this is justifiable if the nucleon wavelength is sufficiently large; nevertheless, the approximation might be too crude for the description of grazing collisions such as those described in the present paper.

\section{CONCLUSIONS}

In conclusion, we have shown that INC/de-excitation models universally fail to describe the cross sections for one-nucleon removal in reactions induced by high-energy protons. This shortcoming is somewhat serious, because INC/de-excitation models are the only viable choice for the description of these observables. We have used simple shellmodel calculations as a guidance for refining the description of the surface in the INC initial conditions. We believe we have presented strong arguments indicating that the reason for this deficiency lies in the presence of neutron skins in heavy, stable nuclei, and in the description of the energy density of the nuclear surface. The refined model, as it is defined here, introduces no fitting parameters and yields encouraging predictions: The one-nucleon-removal cross sections are substantially improved, but are still in disagreement with the experimental values for heavy targets. As a by-product, the isotopic cross sections for the removal of up to several nucleons are also improved by the refined treatment. Still, further work is necessary to achieve closer agreement with the experimental data on heavy nuclei. In the future it will also be necessary to generalize and systematize our approach to any nucleus, magic or nonmagic.

\section{ACKNOWLEDGMENTS}

This work was partially supported by the EU ENSAR Seventh Framework Program project (Grant No. 262010). We wish to thank Dr. José Benlliure for stimulating discussions and for commenting on the manuscript. We express our gratitude to Dr. Stepan Mashnik for his enlightening remarks.

\section{APPENDIX A: DEFINITION OF THE FUZZY ENERGY-RADIUS CORRELATION}

We start by reporting the standard definition of the function that associates the radius of the square potential well to the nucleon kinetic energy in INCL. The original equation [13] [Eq. (4)] is an implicit definition formulated in terms of the nucleon momentum $p$ :

$$
\left(\frac{p}{p_{F}}\right)^{3}=-\frac{4 \pi}{3 A} \int_{0}^{\widetilde{R}(p)} r^{3} \frac{d \rho(r)}{d r} d r
$$

here $p_{F}$ is the Fermi momentum, $A$ is the mass number of the nucleus, and $\rho(r)$ is the assumed space density. We have slightly modified the notation in Ref. [13] to read $\widetilde{R}(p)$. In relation to the function $R(T)$ that we used in the main text of this paper, it should be understood that $R(T)=\widetilde{R}(\sqrt{T(T+2 m)})$. For conciseness' sake, we omit the indication of the nucleon isospin.

\section{Standard algorithm}

The standard INCL algorithm for assigning positions and momenta to a nucleon proceeds as follows:

(i) Draw a random momentum $\vec{p}$ from the uniform Fermi sphere; the vector direction is isotropic and the absolute value is

$$
p=p_{F} u^{1 / 3},
$$

where $u$ is a uniform random number from the $[0,1]$ interval.

(ii) Compute the associated radius $\widetilde{R}(p)$.

(iii) Draw a random position from a uniform sphere of radius $\widetilde{R}(p)$. 
This algorithm trivially results in the following phase-space density [see Fig. 2(a)]:

$$
\frac{d n}{d^{3} \vec{r} d^{3} \vec{p}}=A \frac{\Theta(\widetilde{R}(p)-r)}{(4 \pi / 3) \widetilde{R}(p)^{3}} \frac{\Theta\left(p_{F}-p\right)}{(4 \pi / 3) p_{F}^{3}},
$$

where $\Theta$ represents the Heaviside step function. It was proven in Ref. [13] that Eq. (A1) has the appropriate marginal distributions:

$$
\begin{aligned}
\frac{d n}{d^{3} \vec{r}} & =\int \frac{d n}{d^{3} \vec{r} d^{3} \vec{p}} d^{3} \vec{p}=\rho(r) \\
\frac{d n}{d^{3} \vec{p}} & =\int \frac{d n}{d^{3} \vec{r} d^{3} \vec{p}} d^{3} \vec{r}=A \frac{\Theta\left(p_{F}-p\right)}{(4 \pi / 3) p_{F}^{3}} .
\end{aligned}
$$

\section{Independent algorithm}

The standard algorithm assigns a unique potential radius to nucleons with a given momentum. An extreme alternative would be to make the potential radius completely independent of the momentum. This can be achieved as follows:

(i) Draw a random momentum $p$ from the uniform Fermi sphere:

$$
p=p_{F} u^{1 / 3} .
$$

(ii) Draw another, uncorrelated uniform random number $v$ and define a momentumlike variable $p^{\prime}$ :

$$
p^{\prime}=p_{F} v^{1 / 3} \text {. }
$$

(iii) Use $p^{\prime}$ to compute the potential radius $\widetilde{R}\left(p^{\prime}\right)$.

(iv) Draw a random position from a uniform sphere of radius $\widetilde{R}\left(p^{\prime}\right)$.

It is easy to prove that the independent algorithm yields the following phase-space density,

$$
\frac{d n}{d^{3} \vec{r} d^{3} \vec{p}}=\frac{\rho(r) \Theta\left(p_{F}-p\right)}{(4 \pi / 3) p_{F}^{3}} .
$$

Because Eq. (A3) factorizes in a space part and a momentum part, it is trivial to show that it yields the same marginal distributions as Eq. (A1), viz. Eq. (A2).

\section{Fuzzy algorithm}

The two algorithms above can be seen as limiting cases of the following:

(i) Draw a random momentum $p$ from the uniform Fermi sphere:

$$
p=p_{F} u^{1 / 3} .
$$

(ii) Draw another correlated uniform random number $v$ and define a momentumlike variable $p^{\prime}$ :

$$
p^{\prime}=p_{F} v^{1 / 3} \text {. }
$$

(iii) Use $p^{\prime}$ to compute the potential radius $\widetilde{R}\left(p^{\prime}\right)$.

(iv) Draw a random position from a uniform sphere of radius $\widetilde{R}\left(p^{\prime}\right)$.

The crucial difference with respect to the independent algorithm is that the random numbers $u$ and $v$ are correlated, i.e., they are drawn from some joint distribution function $g(u, v)$.

The phase-space density generated by the fuzzy algorithm is

$$
\frac{d n}{d^{3} \vec{r} d^{3} \vec{p}}=A \frac{\Theta\left(p_{F}-p\right)}{(4 \pi / 3) p_{F}^{3}} \int_{0}^{1} d v g(u, v) \frac{\Theta\left(\widetilde{R}\left(p^{\prime}\right)-r\right)}{(4 \pi / 3) \widetilde{R}\left(p^{\prime}\right)^{3}},
$$

where it is understood that $p$ and $p^{\prime}$ are, respectively, functions of $u$ and $v$ through Eq. (A4). Note that the standard algorithm is recovered for

$$
g(u, v)=\delta(u-v),
$$

while the independent algorithm results from

$$
g(u, v)=1
$$

(remember that $u, v \in[0,1]$ ).

The marginal space and momentum distributions can be shown to be

$$
\begin{aligned}
& \frac{d n}{d^{3} \vec{r}}=-\int_{r}^{\infty} d r^{\prime} \frac{d \rho\left(r^{\prime}\right)}{d r^{\prime}} \cdot g_{v}\left(\left(\frac{\widetilde{R}^{-1}\left(r^{\prime}\right)}{p_{F}}\right)^{3}\right), \\
& \frac{d n}{d^{3} \vec{p}}=A \frac{\Theta\left(p_{F}-p\right)}{(4 \pi / 3) p_{F}^{3}} \cdot g_{u}(u),
\end{aligned}
$$

where $\widetilde{R}^{-1}$ is the inverse function of $\widetilde{R}$ and $g_{u}$ and $g_{v}$ are the marginal distributions of $g$ :

$$
\begin{aligned}
& g_{u}(u)=\int_{0}^{1} d v g(u, v), \\
& g_{v}(v)=\int_{0}^{1} d u g(u, v) .
\end{aligned}
$$

Equation (A6) demonstrates that the fuzzy algorithm generates the appropriate marginal space and momentum distributions [Eq. (A2)] if and only if the marginals of $g$ are uniform:

$$
g_{u}(u)=1, \quad g_{v}(v)=1 .
$$

\section{a. Construction of the joint distribution $g(u, v)$}

Having characterized the conditions for recovering the correct space and momentum distributions, we now show how to construct a joint distribution on the unit square with uniform marginals. We would like the $u-v$ correlation to be continuously "tunable" between the two extreme cases of the standard and the independent algorithm. We therefore introduce a fuzziness parameter $f$ and denote the joint distribution as $g(u, v ; f)$.

There are several solutions to this deceptively simple problem. The one we have adopted in INCL, in a nutshell, consists in generating two correlated normal deviates (which can be done with a simple algorithm) and mapping them to the unit square using the inverse of the normal cumulative distribution function. The method is a simple application of the theory of copulas [43].

In detail, we start out with a bivariate standard normal distribution with correlation coefficient $c$, which can explicitly 
be written as

$$
\begin{gathered}
h(w, z ; c)=\frac{1}{2 \pi \sqrt{1-c^{2}}} \eta(w, z ; c), \\
\eta(w, z ; c)=\exp \left[-\frac{w^{2}+z^{2}-2 c w z}{2\left(1-c^{2}\right)}\right] .
\end{gathered}
$$

Both $w$ and $z$ are standard normal variables:

$$
\begin{aligned}
\int h(w, z ; c) d z & =\frac{1}{\sqrt{2 \pi}} \exp \left(-w^{2} / 2\right), \\
\int h(w, z ; c) d w & =\frac{1}{\sqrt{2 \pi}} \exp \left(-z^{2} / 2\right) .
\end{aligned}
$$

It is easy to show (by factorization) that sampling from $h(w, z ; c)$ can be performed as follows: First sample $w$ from a standard normal distribution, then sample $z$ from a normal distribution with mean $c w$ and variance $1-c^{2}$. We finally define

$$
u=\Phi(w), \quad v=\Phi(z)
$$

where $\Phi(x)$ is the cumulative distribution function of the standard normal distribution. This maps the standard normal random variables $(w, z)$ onto the (open) unit square mapped by $(u, v)$. Because $w$ and $z$ are standard normal variables [Eq. (A7)], $u$ and $v$ are uniform over the unit interval.

The variables $w$ and $z$ are not independent; therefore, neither are $u$ and $v$. However, we were unable to derive a closed expression for the correlation coefficient of $g(u, v ; f)$. We identify the fuzziness parameter $f$ with $1-c$, so that for $c=0$ (uncorrelated variables) we have $f=1$ (full fuzziness) and for $c=1$ (perfect correlation) we have $f=0$ (no fuzziness). The joint distribution can be shown to be

$$
g(u, v ; f)=\left[\sqrt{f(2-f)} \eta\left(\Phi^{-1}(u), \Phi^{-1}(v) ; \frac{1}{1-f}\right)\right]^{-1}
$$

The construction can be straightforwardly generalized to any other initial joint distribution $h(w, z)$ for which a simple sampling algorithm exists.
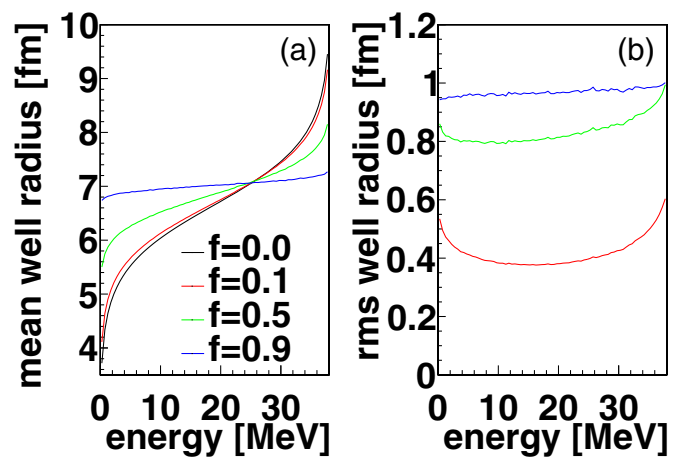

FIG. 11. (Color online) Mean (a) and root-mean-square deviation (b) of the probability distribution for the fuzzy well radius, as functions of the nucleon kinetic energy, for different values of the fuzziness parameter $f$. The rms deviation vanishes for $f=0$ (standard algorithm).

\section{b. Definition of the fuzzy radius}

We conclude by reporting the explicit definition for the function $R(T ; f)$ that we used in the text:

$$
R(T ; f)=\widetilde{R}(\sqrt{T(T+2 m)} ; f) .
$$

Here $\widetilde{R}(p ; f)$ must be understood as a random variable. Based on the description of the fuzzy algorithm above, the probability that $\widetilde{R}(p ; f)$ assumes the value $\xi$, for a given momentum $p$, can be written as

$$
\begin{aligned}
& \frac{d P(\xi<\widetilde{R}(p ; f) \leqslant \xi+d \xi)}{d \xi} \\
& =g(u, v ; f) \frac{d v}{d p^{\prime}} \frac{d p^{\prime}}{d \widetilde{R}\left(p^{\prime}\right)} \\
& =\frac{3 p^{\prime 2}}{\widetilde{R}^{\prime}\left(p^{\prime}\right) p_{F}^{3}} g\left(\left(p / p_{F}\right)^{3},\left(p^{\prime} / p_{F}\right)^{3} ; f\right),
\end{aligned}
$$

where it should be understood that $p^{\prime}=\widetilde{R}^{-1}(\xi ; f)$.

As an illustration, Fig. 11 shows the mean and rootmean-square deviation of the probability distribution given by Eq. (A8), for different values of the fuzziness parameter $f$. It is worth stressing that not only the rms but also the mean values depend on $f$; it is clear that it must be so because for $f=1$ we must recover the independent algorithm, in which the well radius is independent of the nucleon energy.
[1] D. Filges and F. Goldenbaum, Handbook of Spallation Research. Theory, Experiments and Applications (Wiley-VCH, Berlin, 2009).

[2] S. Leray, J.-C. David, M. Khandaker, G. Mank, A. Mengoni, N. Otsuka, D. Filges, F. Gallmeier, A. Konobeyev, and R. Michel, J. Korean Phys. Soc. 59, 791 (2011); http://wwwnds.iaea.org/spallations.

[3] N. P. Jacob and S. S. Markowitz, Phys. Rev. C 11, 541 (1975).

[4] L. Audirac, A. Obertelli, P. Doornenbal et al., Phys. Rev. C 88, 041602 (2013).
[5] D. F. Jackson, Nuclear Reactions (Methuen, London, 1970).

[6] P. Fröbrich, R. Lipperheide, and P. E. Hodgson, Theory of Nuclear Reactions, Oxford Studies in Nuclear Physics (Oxford University Press, Oxford, 1996).

[7] D. Mancusi, A. Boudard, J. Cugnon, J.-C. David, P. Kaitaniemi, and S. Leray, Phys. Rev. C 90, 054602 (2014).

[8] R. Serber, Phys. Rev. 72, 1114 (1947).

[9] L. P. Kadanoff and G. Baym, Quantum Statistical Mechanics: Green's Function Methods in Equilibrium and Nonequilibrium Problems, Frontiers in physics (W. A. Benjamin, San Francisco, 1962). 
[10] V. E. Bunakov and G. V. Matvejev, Z. Phys. A 322, 511 (1985).

[11] A. Boudard, J. Cugnon, J.-C. David, S. Leray, and D. Mancusi, Phys. Rev. C 87, 014606 (2013).

[12] A. Kelić, M. V. Ricciardi, and K.-H. Schmidt, in Joint ICTPIAEA Advanced Workshop on Model Codes for Spallation Reactions, Report INDC(NDC)-0530 (IAEA, Trieste, Italy, 2008), p. 181.

[13] A. Boudard, J. Cugnon, S. Leray, and C. Volant, Phys. Rev. C 66, 044615 (2002).

[14] C.-X. Chen, S. Albergo, Z. Caccia et al., Phys. Rev. C 56, 1536 (1997).

[15] C. Villagrasa-Canton et al., Phys. Rev. C 75, 044603 (2007).

[16] F. Rejmund, B. Mustapha, P. Armbruster et al., Nucl. Phys. A 683, 540 (2001).

[17] L. Giot, J. A. Alcántara-Núñez, J. Benlliure et al., Nucl. Phys. A 899, 116 (2013).

[18] P. Napolitani, K.-H. Schmidt, L. Tassan-Got et al., Phys. Rev. C 76, 064609 (2007).

[19] L. Audouin, L. Tassan-Got, P. Armbruster et al., Nucl. Phys. A 768, 1 (2006).

[20] T. Enqvist, W. Wlazło, P. Armbruster et al., Nucl. Phys. A 686, 481 (2001).

[21] J. Taïeb, K.-H. Schmidt, L. Tassan-Got et al., Nucl. Phys. A 724, 413 (2003).

[22] Y. E. Titarenko et al., Experimental and Theoretical Study of the Yields of Residual Product Nuclei Produced in Thin Targets Irradiated by 100-2600 MeV Protons, INDC report INDC(CCP)-434 (IAEA, Nuclear Data Section, International Nuclear Data Committee, Vienna, Austria, 2002).

[23] R. Michel, M. Gloris, H.-J. Lange et al., Nucl. Instrum. Meth. B 103, 183 (1995).

[24] P. L. Reeder, Phys. Rev. 178, 1795 (1969).

[25] E. A. Remler and A. P. Sathe, Ann. Phys. 91, 295 (1975).

[26] H. Sorge, H. Stöcker, and W. Greiner, Ann. Phys. 192, 266 (1989).

[27] J. Aichelin, Phys. Rep. 202, 233 (1991).
[28] K. Niita, S. Chiba, T. Maruyama, T. Maruyama, H. Takada, T. Fukahori, Y. Nakahara, and A. Iwamoto, Phys. Rev. C 52, 2620 (1995).

[29] J. V. Pilcher, A. A. Cowley, D. M. Whittal, and J. J. Lawrie, Phys. Rev. C 40, 1937 (1989).

[30] S. V. Förtsch, A. A. Cowley, J. J. Lawrie, J. V. Pilcher, F. D. Smit, and D. M. Whittal, Phys. Rev. C 48, 743 (1993).

[31] A. A. Cowley, G. J. Arendse, R. F. Visser et al., Phys. Rev. C 57, 3185 (1998).

[32] R. J. Charity, in Joint ICTP-IAEA Advanced Workshop on Model Codes for Spallation Reactions, report INDC(NDC)-0530 (IAEA, Trieste, Italy, 2008), p. 139; D. Mancusi, R. J. Charity, and J. Cugnon, Phys. Rev. C 82, 044610 (2010).

[33] Y. Yariv and Z. Fraenkel, Phys. Rev. C 20, 2227 (1979).

[34] A. Heikkinen, N. Stepanov, and J. P. Wellisch, in Proceedings to Computing in High Energy and Nuclear Physics 2003 (CHEP03) (SLAC National Accelerator Laboratory, La Jolla, 2003), p. MOMT008; M. Kelsey, D. Wright et al., Nucl. Instrum. Meth. B (to be published).

[35] S. G. Mashnik, K. K. Gudima, R. E. Prael et al., in Joint ICTPIAEA Advanced Workshop on Model Codes for Spallation Reactions, report INDC(NDC)-0530 (IAEA, Trieste, Italy, 2008), p. 51 .

[36] J. Benlliure, K.-H. Schmidt, D. Cortina-Gil et al., Nucl. Phys. A 660, 87 (1999).

[37] J. Zenihiro, H. Sakaguchi, T. Murakami et al., Phys. Rev. C 82, 044611 (2010).

[38] J. Blomqvist and S. Wahlborn, Ark. Fys. 16, 545 (1960).

[39] E. Friedman and A. Gal, Phys. Rep. 452, 89 (2007).

[40] A. Kelić, K.-H. Schmidt, T. Enqvist et al., Phys. Rev. C 70, 064608 (2004).

[41] S. Leray et al., Phys. Rev. C 65, 044621 (2002).

[42] This will anyway be necessary if we wish to generalize our treatment to nonmagic nuclei.

[43] R. B. Nelsen, An Introduction to Copulas (Springer, New York, 1999). 